\title{
Mathematical modeling of the electron- beam wire deposition additive manufacturing by the smoothed particle hydrodynamics method
}

\author{
Dmitriy Nikolayevich Trushnikov', Elena Georgieva Koleva ${ }^{2,3,4}$, Roman Pozolovich Davlyatshin?', \\ Roman Mikhailovich Gerasimov ${ }^{1}$ and Yuriy Vitalievich Bayandin ${ }^{1,5^{*}}$ (D)
}

\begin{abstract}
Background: The actual problem for calculating a shape of free surface of the melt when analyzing the processes of wire-based electron-beam surfacing on the substrate, being introduced into additive manufacturing, is the development of adequate mathematical models of heat and mass transfer. The paper proposed a formulation of the problem of melt motion in the framework of the Lagrangian description. The mathematical statement includes the balance equations for mass, momentum and energy, and physical equations for describing heat and mass transfer.

Methods: The smoothed particle hydrodynamics method was used for numerical simulation of the process of wirebased electron-beam surfacing on the substrate made from same materials (titanium or steel). A finite-difference analog of the equations is given and the algorithm for solving the problem is implemented. To integrate the discretized equations Verlet method was utilized. Algorithms are implemented in the open software package LAMMPS.

Results: The numerical simulation results allow the estimation of non-stationary volume temperature distributions, melt flow velocities and pressures, and characteristics of process.
\end{abstract}

Conclusion: The possibility of applying the developed mathematical model to describe additive production is shown. The comparison of numerical calculations with experimental studies showed good agreement.

Keywords: Additive manufacturing, Electron-beam wire surfacing, Steel, Titanium, Numerical simulation, Smoothed particle hydrodynamics, Heat-and-mass transfer

\section{Introduction}

Today, it is important to ensure such conditions where we can apply the digital manufacturing concept to aerospace enterprises. The digital manufacturing concept (Industrial 4.0) calls for the integration of industry and digital technologies to create digital manufacturing facilities or smart plants and factories where all devices, machines, products and people communicate by means of digital technologies. The fact that hybrid technologies are fast-evolving leads to the necessity of multivariant

\footnotetext{
* Correspondence: buv@icmm.ru

'Perm National Research Polytechnic University, 29 Komsomolsky prospekt,

Perm, Russian Federation 614990

${ }^{5}$ Institute of Continuous Media Mechanics UB RAS, 1 Ac. Korolev str, Perm,

Russian Federation 614013

Full list of author information is available at the end of the article
}

simulation modeling for manufacturing processes, which makes it possible to preliminarily select technological parameters for various product manufacturing processes, including the build-up process, as well as considerably reduce the technology development stage.

The technology of electron-beam wire additive manufacturing (Lorenz et al. 2015; Ding et al. 2015; Stawovy 2018; Fuchs et al. 2018; Fortuna et al. 2018; Tarasov et al. 2018) uses an electron beam to melt wire filler material. In the process of layer-by-layer melting, a workpiece is manufactured for subsequent processing. The vacuum necessary for the implementation of the electron beam welding process also provides exceptional protection of molten metal during a workpiece manufacturing. The use of filler wire as a starting material 
eliminates the problems associated with a low productivity of existing methods, a high cost of equipment, limited types of materials used associated with the traditional use of powder systems fused with a powerful heat source as the initial material for the additive formation (Jhavar et al. 2014; Kapil et al. 2016; Zhang et al. 2017). Wire materials are easier to store, they create fewer safety and health problems compared to metal powders (especially titanium, magnesium alloys and other active metals). The manufacturing productivity of parts from powder materials in traditional additive technologies is extremely low, which virtually prevents the use of these technologies for production of large-sized items. The technology of hybrid manufacturing of products combines the best characteristics of the additive manufacturing and the subsequent mechanical removal of a material in the process of creating metal products (Kapil et al. 2016; Zhang et al. 2017). The process can be implemented on one platform with a hybrid layer-by-layer deposition of a wire material and processing using a $\mathrm{CNC}$ machine and is optimal to manufacture large-sized parts of with a low and medium complexity. An important advantage is that the wires are manufactured for the welding industry and are available in a wide range.

It's very difficult to manage the process of additive manufacturing of wire materials. Transverse dimensions and shape of a bead, the ongoing metallurgical processes and the formed structure of the material will depend on many factors, most of which cannot be completely controlled. In the framework of the concept of the digital manufacturing, the manufacturing stage of a product is preceded by a preliminary simulation of a digital prototype behavior under a given set of factors in order to determine a product parameters or its manufacturing technology to ensure the required operational characteristics.

Additive manufacturing (AM) has much in common with welding and related processes. A moving heat source forms a weld pool that moves with the heat source. From a technological point of view, additive technologies are closest to multilayer surfacing, in which the source material (filler wire) also interacts with the heat source, layers grow gradually, thermal cycles are superimposed as new layers are added, and transitional changes in geometry are some of the features that must be taken into account for a better understanding of AM. A complete statement requires solving the related problem of heat conduction and melt flow (Markl and Körner 2016). The density and other thermophysical characteristics of the material in the solid and liquid state are often considered constant, since this assumption saves computational time. Thermal effects associated with the evaporation of alloying elements are also ignored, since the effect is usually small compared to the input energy from the heat source.
There are attempts to analytically solve the heat problem to describe processes in additive manufacturing (Fathi et al. 2006; Huang et al. 2016; Mladenov et al. 2018). An adequate description of an arbitrary geometry requires a solution using numerical methods. The solution to the thermal problem of finite element methods for predicting the size of the weld pool is proposed in (Soylemez et al. 2010). Heat transfer and fluid flow models using finite difference method (FDM) are considered in the works of (Jamshidinia et al. 2013; Yuan and $\mathrm{Gu}$ 2015). To describe the motion of the free surface, the Level-Set method in a combination with the Volume of fluid (VOF) using the finite difference methods (FDM) are used (He and Mazumder 2007; Morville et al. 2012; Qi et al. 2006; Lee et al. 2014; Lee and Zhang 2015) The considered modeling methods allow us to describe in detail the processes of heat and mass transfer in the additive manufacturing of products by melting wire materials with a detailed account of the behavior dynamics of the free surface of the metal melt. The results of such modeling are effective to understand the physical features of the processes, but they need significant computational costs. The application of the results may be more in obtaining general criteria relations $(\mathrm{Hu}$ et al. 2019), rather than to predict the geometric parameters of the deposited beads. The use of calculations to predict the geometric parameters of the weld bead requires the search for high-performance calculation methods. The application of the SPH method looks promising. This method is highly productive, effective in organizing parallel computing, and is used in particular in computer simulators to simulate the flow of a fluid with a free surface in real time. There appear first works on the application of this method in modeling welding processes ( $\mathrm{Hu}$ and Eberhard 2017; Trautmann et al. 2018) or in modeling powder-bed additive processes (Russell et al. 2018). At the same time, this method is practically not mentioned in the open media about the use of this method to model the wire-based additive manufacturing. In the present paper, we propose a description of the numerical implementation using the $\mathrm{SPH}$ method for modeling the electron-beam wire additive manufacturing. The numerical implementation was carried out using open-source application software LAMMPS, which may be of a particular interest to readers.

\section{Smoothed particle hydrodynamics method}

In addition to grid-based and finite element methods of numerical modeling for the motion, deformation and destruction of condensed media, there are mesh-free methods (Liu 2009) that, in some cases, are more effective and less strict with regard to computational resources, one of which is the "smoothed particle 
hydrodynamics" method (SPH) independently suggested by several authors in 1977, initially for the purpose of solving problems in astrophysics (Lucy 1977; Gingold and Monaghan 1977) and later for the purpose of solving problems in continuum mechanics (Johnson et al. 1996; Libersky and Petschek 1991; Libersky et al. 1993). For the sake of convenience, the abbreviation SPH will be used to specify the name of this numerical method.

As for mesh-free methods, there is no need to determine mesh nodes or elements as, for example, in case of finite element methods. In order to describe the condition of a continuous medium (a solid, fluid or gas), the SPH method needs a certain set of points known as particles or pseudo-particles. For the purpose of describing the evolution of a medium, the SPH method uses an absolutely Lagrangian approach that permits the discretization of a prescribed set of continuum equations by interpolating the properties directly at a discrete set of points distributed over the solution domain with no need to define a spatial mesh. The method's Lagrangian nature associated with the absence of a fixed mesh is its main strength. Difficulties associated with the motion of a medium and structural problems involving large deformations and free surfaces are resolved in a natural way.

At its core, the SPH method can be regarded as an intuitive discretization method for continuum partial differential equations. In this respect, the SPH method is quite similar to the finite element method, but unlike the latter, uses an evolving interpolation scheme to approximate a field variable at any point in a domain. The value of a variable at a particle of interest can be approximated by summing the contributions from a set of neighboring particles whose contribution is defined by a functional kernel that plays a smoothing role for field variables at a certain specific scale.

The SPH method is effective to apply to processes with large deformations (Monaghan 2006; Ba and Gakwaya 2018). The method's most popular applications are as follows: fluid spraying, wave dynamics, ballistics, spraying (as in paint spraying), gas flow, fragmentation and destruction. There are many applications for which both the coupled Eulerian-Lagrangian and the SPH methods can be used. In many coupled EulerianLagrangian approaches the material's void ratio is high and, consequently, computational efforts may be prohibitively high. In these cases, the SPH method is preferred. For example, tracking fragments from a primary effect until a secondary effect occurs can be very expensive in a coupled Eulerian-Lagrangian analysis but comes at no additional cost in SPH. The SPH methods' effective application is well confirmed in impact effect and fracture problems. The studies by Johnson, Stryk and Beissel (Johnson et al. 1996), Hayhurst and Clegg
(Hayhurst and Clegg 1997) (these two authors implemented the method into the AUTODYN-2D commercial application package for dynamic modeling) were among the first to illustrate the SPH application for modeling a high-velocity dynamic effect. Modern application packages, such as ABAQUS/Explicit (Ba and Gakwaya 2018; Zahedi et al. 2013; Demiral 2014) and ANSYS/LSDYNA (Liu et al. 2017; Terranova et al. 2018), allow us to carry out the numerical modeling of dynamic processes using the SPH method, including in a coupled setting when bodies are represented by various heterogeneous media, some of which are modeled by the finite element approach and others by the SPH method (Xiao et al. 2011). As is evident from the foregoing, the SPH method is rather widely used to describe the motion of fluids (Liu 2009; Monaghan 2006; Morris et al. 1997).

The method's application to the mechanics of solids is still a rather unexplored area, but recently there has been an intensive growth in metal treatment (Bonet and Kulasegaram 2000; Ma and Hartmaier 2016; Xiao et al. 2017; Stenberg et al. 2017; Spreng and Eberhard 2018; Zhang et al. 2019) and melting/crystallization (Monaghan et al. 2005; Faizal and Septiawan 2014; Farrokhpanah et al. 2017; Cleary 2010; Cleary et al. 2002; Kulasegaram et al. 2003; Alexiadis et al. 2018; Gerasimov et al. 2017) works. When modeling mechanical processes in solids, researchers often use traditional isotropic von Mises (Bonet and Kulasegaram 2000; Benz and Asphaug 1995; Batra and Zhang 2004; Cleary and Das 2008) and Johnson-Cook (Johnson and Cook 1985; Batra and Zhang 2008; Gong et al. 2015; Dong et al. 2017; Dong et al. 2018) plasticity models that proved themselves to be good for dynamic and impact loadings. However, questions remain unanswered when it comes to the description, with the use of the SPH method, of the anisotropy of physicomechanical properties of metals and alloys under thermomechanical effect, melting and crystallization.

$>$ The thermomechanical treatment of metals and alloys in both fluid and solid phases can cause various perturbations resulting in the fact that a favorable mode becomes unstable. The modeling of the perturbation evolution in the SPH method is a topical and largely unexplored problem (Rahmat et al. 2014; Shadloo et al. 2013; Shadloo and Yildiz 2011; Shadloo and Yildiz 2012).

\section{Approximation of heat and mass transfer equations by the SPH method}

The differential equation system that describes the evolution of density $\rho$, velocity $\vec{u}$ and specific energy $e$ of a continuous medium in the form of balance laws is under consideration. 


$$
\left(\begin{array}{l}
\frac{d \rho}{d t}=-\rho \nabla \cdot \vec{u}, \\
\frac{d \vec{u}}{d t}=\frac{1}{\rho} \nabla \cdot \overline{\bar{\sigma}}, \\
\frac{d e}{d t}=\frac{1}{\rho} \overline{\bar{\sigma}}: \overline{\bar{\tau}}-\frac{1}{\rho} \nabla \cdot \vec{q}, \\
\overline{\bar{\sigma}}=-p \overline{\bar{I}}+\overline{\bar{\tau}}, \\
\tau_{i j}=\mu\left(\frac{\partial u_{i}}{\partial x_{j}}+\frac{\partial u_{j}}{\partial x_{i}}\right), \\
\vec{q}=-\lambda \nabla T,
\end{array}\right.
$$

where $\vec{u}=d \vec{r} / d t$ - velocity, $\vec{r}-$ position vector of a particle, $\rho$ - density, $\lambda$ - thermal conductivity coefficient, $\vec{q}-$ thermal flow, $T-$ temperature, $\overline{\bar{\sigma}}-$ stress tensor, $\overline{\bar{I}}-$ unit tensor, $p$ - pressure, $\mu$ - viscosity, $\overline{\bar{\tau}}-$ viscous stress tensor.

The SPH method's principle consists in approximating field variables $\{\rho, \vec{u}, e, \overline{\bar{\sigma}}, \vec{q}\}$. The transition from a continual medium to a discrete one implies that continuous function $f(\vec{r})$ that characterizes a field variable is replaced by its discrete analog $f_{i}$. Piecewise constant value $f_{i}$ that is determined for each particle $i$ as amount $N$ of values $f_{j}$ from particles $j$ of the circumference within prescribed distance $h$

$$
f_{i}=\sum_{j=1}^{N} m_{j} \frac{f_{j}}{\rho_{j}} W\left(\left\|\vec{r}_{i}-\vec{r}_{j}\right\|\right)=\sum_{j=1}^{N} m_{j} \frac{f_{j}}{\rho_{j}} W_{i j},
$$

where $m_{j}$ - mass of a particle, $\rho_{j}$ - density of a particle, $W$ - smoothing function (kernel).

In (2) and further in the text we use equivalent designations $W\left(\left\|\vec{r}_{i}-\vec{r}_{j}\right\|\right)=W\left(\left\|\vec{r}_{i j}\right\|\right)=W_{i j}$ where $\vec{r}_{i}-\vec{r}_{j}$ $=\vec{r}_{i j}$. Formally, in expression (2) the summation takes place only for particles inside a certain domain for which $\left\|\vec{r}_{i}-\vec{r}_{j}\right\|<h$. The volume integration procedure allows us to turn from partial differential eq. (1) to ordinary differential equations.

In this case the SPH method's advantage is that coordinate derivatives affect neither the mass of particles $m_{j}$ nor field values $f_{j}$, and the differentiation takes place only for weight functions $W_{i j}$. At the same time, the gradient of a certain value can be presented as follows

$$
\begin{aligned}
\nabla f_{i} & =\sum_{j=1}^{N} m_{j} \frac{f_{j}}{\rho_{j}} \nabla_{j} W\left(\left\|\vec{r}_{i}-\vec{r}_{j}\right\|\right) \\
& =\sum_{j=1}^{N} m_{j} \frac{f_{j}}{\rho_{j}} \nabla_{j} W_{i j}
\end{aligned}
$$

Let us consider the properties of weight function $W$ (kernel). The kernel is subject to a compactness (normalization) limitation

$$
\int W(\|\vec{r}\|, h) d \vec{r}=1
$$

At $h \rightarrow 0$, the kernel turns into $\delta$-function

$$
\lim _{h \rightarrow 0} W(\|\vec{r}\|, h)=\delta(\|\vec{r}\|) .
$$

The radial symmetry of function $W$ makes it possible to turn from the gradient to its derivative as follows

$$
\nabla_{j} W_{i j}=\frac{d W}{d r} \frac{\vec{r}_{i}-\vec{r}_{j}}{\left\|\vec{r}_{i}-\vec{r}_{j}\right\|}=\frac{d W}{d r} \frac{\vec{r}_{i j}}{\left\|r_{i j}\right\|}
$$

Property (6) implies the anti-symmetry of gradient $\nabla_{j} W_{i j}=-\nabla_{j} W_{j i}$. It is also easy to note that an expression for the density of particles is obtained by averaging the mass for weight functions

$$
\rho_{i}=\sum_{j=1}^{N} m_{j} W_{i j}
$$

It should be noted that expression (7) implies a certain average mass in radius domain $\left\|\vec{r}_{i}-\vec{r}_{j}\right\|<h$. Whereas the remaining field values will have the weighed averaging.

Before formulating a full statement of hydrodynamics equations (Navier-Stokes equations), additional expressions for differentiation operators will be required. Let us consider the continuity equation (the first one from (1)) that contains the velocity vector's divergence. For the differentiation operator we will use the property

$$
\nabla \cdot(\rho \vec{u})=\rho \nabla \cdot \vec{u}+\vec{u} \cdot \nabla \rho,
$$

that allows us to rewrite the continuity equation as follows

$$
\frac{d \rho}{d t}=\nabla \cdot(\rho \vec{u})-\vec{u} \cdot \nabla \rho .
$$

By applying the smoothed particle method's discretization and expression (3) to continuity eq. (9) we will obtain

$$
\begin{gathered}
\frac{d \rho_{i}}{d t}=\sum_{j=1}^{N} m_{j} \vec{u}_{j} \cdot \nabla_{j} W_{i j}-\vec{u}_{i} \cdot \sum_{j=1}^{N} m_{j} \nabla_{j} W_{i j}=-\sum_{j=1}^{N} m_{j}\left(\vec{u}_{i}-\vec{u}_{j}\right) \cdot \nabla_{j} W_{i j}= \\
=-\sum_{j=1}^{N} m_{j} \vec{u}_{i j} \cdot \nabla_{j} W_{i j} .
\end{gathered}
$$

In order to derive the momentum balance equation's discretization (the second one from (1)), we will use the stress tensor's divergence 


$$
\nabla \cdot \frac{\overline{\bar{\sigma}}}{\rho}=-\frac{\overline{\bar{\sigma}}}{\rho^{2}} \nabla \rho+\frac{1}{\rho} \nabla \cdot \overline{\bar{\sigma}} .
$$

By expressing the last addend from (11) and inserting it into momentum balance eq. (1) we will obtain

$$
\frac{d \vec{u}}{d t}=\frac{\overline{\bar{\sigma}}}{\rho^{2}} \cdot \nabla \rho+\nabla \cdot \frac{\overline{\bar{\sigma}}}{\rho} .
$$

The spatial derivatives in (12) can be also represented with the use of expression (3)

$$
\begin{aligned}
& \nabla \rho=\sum_{j=1}^{N} m_{j} \nabla_{j} W_{i j}, \\
& \nabla \cdot \frac{\overline{\bar{\sigma}}}{\rho}=\sum_{j=1}^{N} m_{j} \frac{\overline{\bar{\sigma}}_{j}}{\rho_{j}^{2}} \cdot \nabla_{j} W_{i j} .
\end{aligned}
$$

The final motion equation for particle $i$ will be written as

$$
\frac{d \vec{u}_{i}}{d t}=\frac{\overline{\bar{\sigma}}_{i}}{\rho_{i}^{2}} \cdot \sum_{j=1}^{N} m_{j} \nabla_{j} W_{i j}+\sum_{j=1}^{N} m_{j} \frac{\overline{\bar{\sigma}}_{j}}{\rho_{j}^{2}} \cdot \nabla_{j} W_{i j},
$$

that, taking into account multiplier $m_{i}$, leads to an expression for the force that affects particle $i$

$$
\vec{f}_{i}=m_{i} \frac{d \vec{u}_{i}}{d t}=\sum_{j=1}^{N} m_{i} m_{j}\left(\frac{\overline{\bar{\sigma}}_{i}}{\rho_{i}^{2}}+\frac{\overline{\bar{\sigma}}_{j}}{\rho_{j}^{2}}\right) \cdot \nabla_{j} W_{i j} .
$$

It is obvious that the expression for force (16) is antisymmetric due to (6). From there it follows that this discretization preserves a total momentum.

Similarly, an equation is derived for the balance of specific energy

$$
\begin{aligned}
m_{i} \frac{d e_{i}}{d t}= & \frac{1}{2} \sum_{j=1}^{N} m_{i} m_{j}\left(\frac{\overline{\bar{\sigma}}_{i}}{\rho_{i}^{2}}+\frac{\overline{\bar{\sigma}}_{j}}{\rho_{j}^{2}}\right): \vec{u}_{i j} \nabla_{j} W_{i j} \\
& +\sum_{j=1}^{N} \frac{m_{i} m_{j}}{\rho_{i} \rho_{j}} \frac{\left(\lambda_{i}+\lambda_{j}\right)\left(T_{i}-T_{j}\right)}{r_{i j}^{2}} \vec{r}_{i j} \cdot \nabla_{j} W_{i j} .
\end{aligned}
$$

For a viscous medium, we use the closing equation (defining a ratio) that connects the stress tensor with the velocity gradient as follows

$$
\overline{\bar{\sigma}}=-p I+\overline{\bar{\tau}}, \quad \tau_{i j}=\mu\left(\frac{\partial u_{i}}{\partial x_{j}}+\frac{\partial u_{j}}{\partial x_{i}}\right) .
$$

Closing eq. (18) makes it possible to represent the motion equation's right part using the discretization similar to the representation of thermal flows and suggested in work (Morris et al. 1997).

$$
\frac{1}{\rho} \nabla \cdot \mu \nabla \vec{u}=\sum_{j=1}^{N} \frac{m_{j}\left(\mu_{i}+\mu_{j}\right)}{\rho_{i} \rho_{j}} \frac{\vec{r}_{i j}}{r_{i j}^{2}} \cdot \nabla_{j} W_{i j} \vec{u}_{i j} .
$$

The representation of viscous addend (19) is allowed to write the discrete analog of the force that affects particle $i$ (motion equation) as follows

$$
\begin{aligned}
\vec{f}_{i}=m_{i} \frac{d \vec{u}_{i}}{d t}= & -\frac{1}{2} \sum_{j=1}^{N} m_{i} m_{j}\left(\frac{p_{i}}{\rho_{i}^{2}}+\frac{p_{j}}{\rho_{j}^{2}}\right) \nabla_{j} W_{i j} \\
& +\sum_{j=1}^{N} \frac{m_{i} m_{j}\left(\mu_{i}+\mu_{j}\right) \vec{u}_{i j}}{\rho_{i} \rho_{j}}\left(\frac{1}{r_{i j}} \frac{\partial W}{\partial r}\right) .
\end{aligned}
$$

In order to correctly describe dissipative addends in an energy equation, it is necessary to consider the volumetric and viscous components of stress tensor (18)

$$
\begin{gathered}
m_{i} \frac{d e_{i}}{d t}=-\frac{1}{2} \sum_{j=1}^{N} m_{i} m_{j}\left(\frac{p_{i}}{\rho_{i}^{2}}+\frac{p_{j}}{\rho_{j}^{2}}\right) \vec{u}_{i j} \cdot \nabla_{j} W_{i j}+ \\
+\frac{1}{2} \sum_{j=1}^{N} \frac{m_{i} m_{j}\left(\mu_{i}+\mu_{j}\right)}{\rho_{i} \rho_{j}}\left(\frac{1}{r_{i j}} \frac{\partial W}{\partial r}\right) \vec{u}_{i j} \cdot \vec{u}_{i j}+\sum_{j=1}^{N} \frac{m_{i} m_{j}}{\rho_{i} \rho_{j}} \frac{\left(\lambda_{i}+\lambda_{j}\right)\left(T_{i}-T_{j}\right)}{r_{i j}^{2}} \vec{r}_{i j} \cdot \nabla_{j} W_{i j} \cdot
\end{gathered}
$$

Equations (20-21) represent a formulation suitable for computations by the SPH method. Such realization is presented in the LAMMPS open package (a particle modeling code developed and supported at Sandia National Laboratories, USA) (Ganzenmuller et al. 2011). This package is first of all intended for atomistic modeling, but also gives a general, completely parallelized basis for particle modeling based on Newton's motion equations. In addition to a full system of equations for density (10), velocity (20) and energy (21), there is a closing equation for temperature $T=e / C_{v}$ where $C_{v}$-specific thermal capacity. The statement is complemented by initial conditions and thermally insulated boundary conditions (i.e. zero heat flux) that are set for nodes (SPH particles). The power of the electron beam and the model heat source are connected as follows: the power of the electron beam is multiplied by the integration step, then the resulting energy is evenly divided into particles entering the electron beam.

Note that for the description of phase transformations, all particles that are lower than the melting temperature are fixed. The viscosity in the liquid and solid phase is a single constant, which is a fairly common technique, since particles below the melting point are frozen. Besides, accounting for capillary forces in models of this type is necessary. However, at the first stages of work, we assess the possibilities of using $\mathrm{SPH}$ without taking into account capillary force models. This is done for high performance source code. 


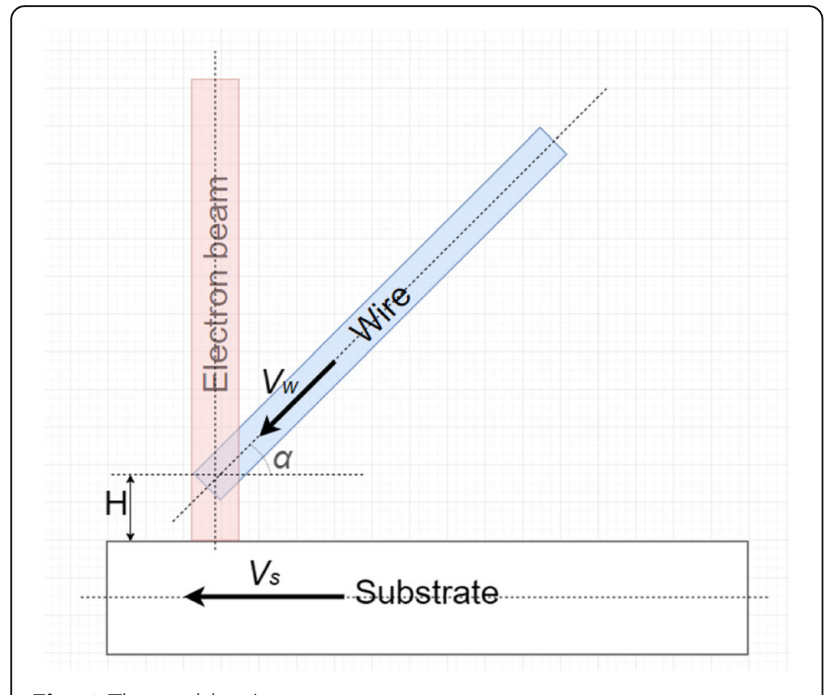

Fig. 1 The problem's geometry

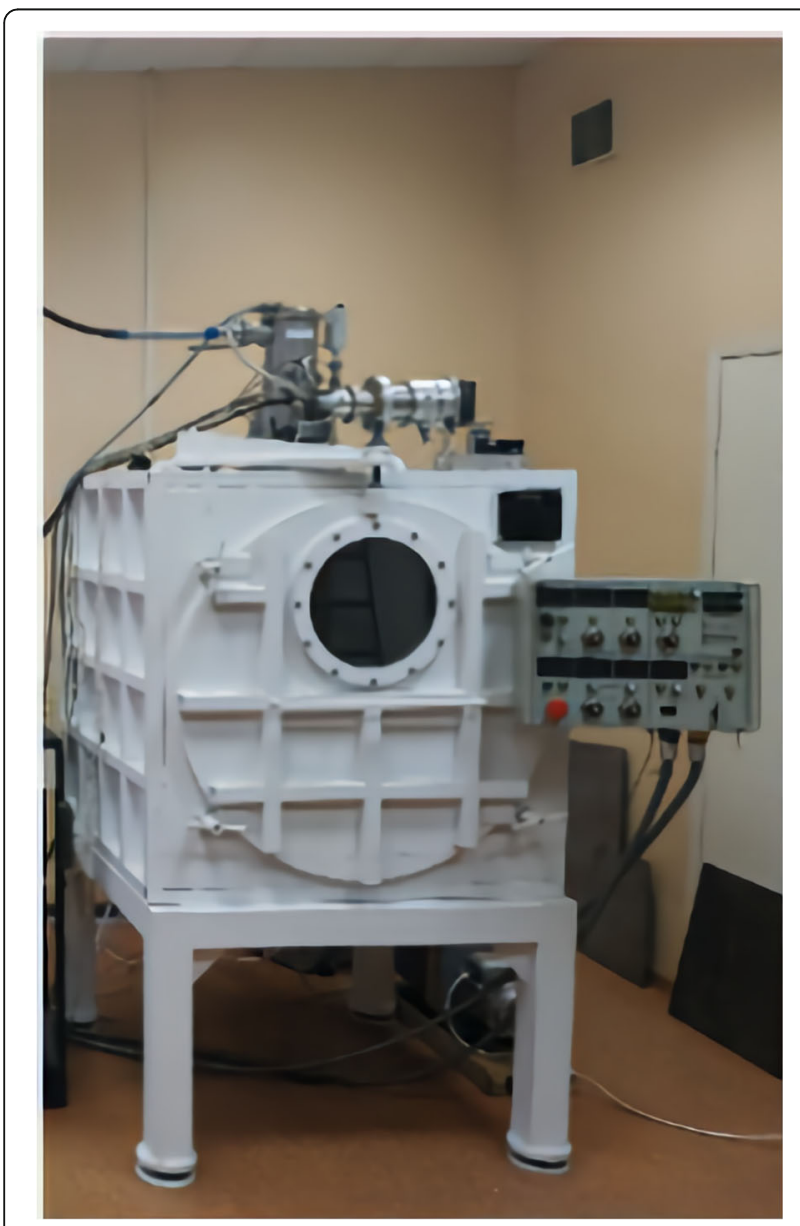

Fig. 2 The modernized installation for additive layer-by-layer product formation by an electron beam
When integrating obtained differential equations for viscous flows, a time step is limited by a viscous component, unlike the well-known Courant condition (for example, as in grid-based methods). Therefore, stable numerical modeling should be carried out with the time step

$$
\Delta t<0,125 \frac{h^{2}}{v},
$$

where $h$-discretization parameter of the SPH method, $v=\mu / \rho-$ kinematic viscosity.

The LAMMPS code realizes a standard numerical scheme of Verlet integration that consists in the sequential algorithm used to calculate particle velocity values (Ganzenmuller et al. 2011):

1a) determination of particle velocities $\vec{u}_{i}\left(t+\frac{1}{2} \Delta t\right)$

$=\vec{u}_{i}(t)+\frac{\Delta t}{2 m_{i}} \vec{f}_{i}(t)$

2b) determination of particle positions $\vec{r}_{i}(t+\Delta t)$

$=\vec{r}_{i}(t)+\Delta t \vec{u}_{i}\left(t+\frac{1}{2} \bowtie t\right)$

3) determination of forces $\vec{f}_{i}(t+\Delta t)$ as per eq. (20)

4) determination of particle velocities for the end of a step $\vec{u}_{i}(t+\Delta t)=\vec{u}_{i}\left(t+\frac{1}{2} \Delta t\right)+\frac{\Delta t}{2 m_{i}} \vec{f}_{i}(t+\Delta t)$

The above integration scheme cannot directly apply to the SPH method because forces are calculated based on velocities determined in a half time step. This results in violating the balance of mass, momentum and energy as equations $(10,20)$ and (21) obviously depend on the particle velocities. This situation can be improved by calculating the particle velocities extrapolated to a full step

$$
\widetilde{\vec{u}}_{i}(t+\Delta t)=\vec{u}_{i}(t)+\frac{\Delta t}{m_{i}} \vec{f}_{i}(t) .
$$

By adding the integration of equations for the local density and internal energy we will write the full Verlet scheme as follows (Ganzenmuller et al. 2011):

1a) $\vec{u}_{i}\left(t+\frac{1}{2} \Delta t\right)=\vec{u}_{i}(t)+\frac{\Delta t}{2 m_{i}} \vec{f}_{i}(t)$;

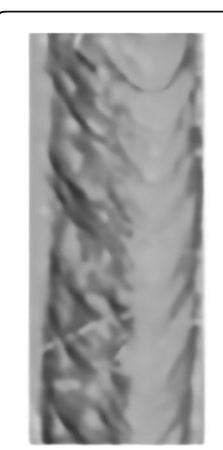

1

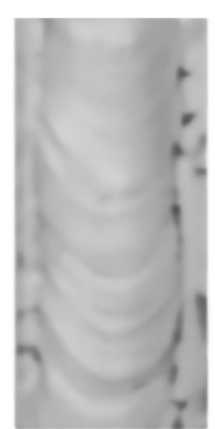

2

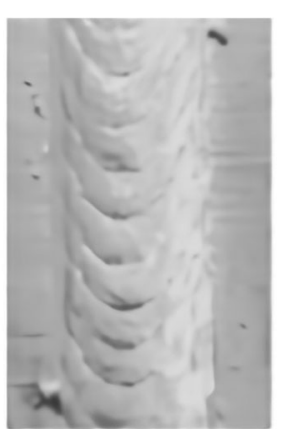

3
Fig. 3 Finished weld beads with mode numbers 
Table 1 Geometrical characteristics of the bead

\begin{tabular}{|c|c|c|c|c|c|c|}
\hline \multirow[t]{2}{*}{ Mode No. } & \multirow[t]{2}{*}{ Bead width, [mm] } & \multirow[t]{2}{*}{ Bead height, [mm] } & \multirow{2}{*}{$\begin{array}{l}\text { Estimated bead } \\
\text { width, [mm] }\end{array}$} & \multirow{2}{*}{$\begin{array}{l}\text { Estimated bead } \\
\text { height, }[\mathrm{mm}]\end{array}$} & \multicolumn{2}{|c|}{ Relative error, \% } \\
\hline & & & & & Width & Height \\
\hline 1 & 3.3 & 1 & 3.45 & 0.87 & 4 & 5 \\
\hline 2 & 3.6 & 1 & 3.7 & 0.9 & 3 & 3 \\
\hline 3 & 3.6 & 0.7 & 3.8 & 0.6 & 3 & 3 \\
\hline
\end{tabular}

1b) $\widetilde{\vec{u}}_{i}(t+\Delta t)=\vec{u}_{i}(t)+\frac{\Delta t}{m_{\dot{d}}} \vec{f}_{i}(t)$;

1c) $\rho_{i}\left(t+\frac{1}{2} \Delta t\right)=\rho_{i}(t)+\frac{\Delta t}{2} \frac{d \rho_{i}}{d t}(t)$;

1d) $e_{i}\left(t+\frac{1}{2} \Delta t\right)=e_{i}(t)+\frac{\Delta t}{2} \frac{d e_{i}}{d t}(t)$

1e) $\vec{r}_{i}(t+\Delta t)=\vec{r}_{i}(t)+\Delta t \vec{u}_{i}\left(t+\frac{1}{2} \Delta t\right)$;

2) determination of right parts $\vec{f}_{i}(t+\Delta t), \frac{d \rho_{i}}{d t}(t+\Delta t)$, $\frac{d e_{i}}{d t}(t+\Delta t)$ at the end of a time step;

3a) $\rho_{i}(t+\Delta t)=\rho_{i}\left(t+\frac{1}{2} \Delta t\right)+\frac{\Delta t}{2} \frac{d \rho_{i}}{d t}(t+\Delta t)$;

3b) $e_{i}(t+\Delta t)=e_{i}\left(t+\frac{1}{2} \Delta t\right)+\frac{\Delta t}{2} \frac{d e_{i}}{d t}(t+\Delta t)$;

3c) $\vec{u}_{i}(t+\Delta t)=\vec{u}_{i}\left(t+\frac{1}{2} \Delta t\right)+\frac{\Delta t}{2 m_{i}} \vec{f}_{i}(t+\Delta t)$.

The division of the evolution in time for the density and energy into two separate steps gives, similarly to the integration of velocity $\vec{u}$, substantial advantages with regard to the simple Euler scheme for $\rho$ and $e$ that, as we know, leads to the violation of balance equations.

A computation algorithm specific to the LAMMPS package includes the following steps (Ganzenmuller et al. 2011):

1) Setting the initial distribution of particles.

2) Setting initial parameters for a problem

(thermophysical characteristics, particle velocities, a time integration step).

3) A time cycle that realizes a simultaneous solution for heat and mass transfer equations at each time iteration.

4) Setting external effects (gravitation, thermal source) at each iteration. Gravitational component $\vec{g}$ is added to eq. (20). And external thermal flow $\vec{q}$ to eq. (21).

5) Saving computation results.

\section{Problem statement of wire surfacing}

The problem of wire build-up (Grade 2 Titanium and 308LSi Steel) onto a substrate from the same material in the spray transfer mode, with the low power of a

Table 2 Physical characteristics of the Grade 2 Titatium and the 308LSi Steel

\begin{tabular}{llll}
\hline Property & Designation & Grade 2 & 308LSi \\
\hline Density & $\rho\left[\mathrm{kg} / \mathrm{m}^{3}\right]$ & 4510 & 7680 \\
Melting temperature & $T[\mathrm{~K}]$ & 1941 & 1732 \\
Thermal conductivity & $\lambda[\mathrm{W} /(\mathrm{m} \cdot \mathrm{K})]$ & 16.76 & 26 \\
Thermal capacity & $\left.C_{V}[\mathrm{~J} / \mathrm{K} \cdot \mathrm{kg})\right]$ & 700 & 710 \\
Dynamic viscosity & $\mu[\mathrm{Pa} \cdot \mathrm{s}]$ & 0.01 & 0.007 \\
\hline
\end{tabular}

thermal source, is under consideration. The problem's geometry is presented in Fig. 1.

For the purpose of modeling heat and mass transfer processes with the wire material build-up, we selected the following variable parameters:

1) power of a thermal source;

2) distribution of the density of a thermal source's energy flow;

3) initial temperature of a sample (300 K);

4) power and distribution of an additional volume source (in case of additional induction heating);

5) dependence of a material's thermophysical characteristics on temperature;

6) characteristics of phase transitions;

7) substrate displacement velocity $V_{s}$;

8) wire feed rate $V_{w}$;

9) wire feed angle $\alpha$;

10) wire height above the substrate $H$.

\section{Determination of the geometry and characteristics of finished weld beads in natural experiments}

When carrying out experimental works, we used the ELA-6VCh electron-beam energy system that creates an electron beam of up to $6 \mathrm{~kW}$ and a designed and manufactured installation for additive product formation that includes a vacuum chamber of $1 \mathrm{~m}^{3}$ with a product positioning mechanism and a wire feed system with differential vacuum pumping (Fig. 2).

The quality of bead formation under electron beam building up depends on how a heating area covers a welding wire material's surface. This requires either the electron beam's defocusing in the wire end's heating area or the electron beam's scanning that is realized by giving variable signals of a prescribed form to the electron beam gun's deflecting system. The electron beam's defocusing leads to a decrease in its specific power in the heating zone, therefore the use of the beam's scanning is more preferable. The experimental studies under discussion used the scanning along the arc of a circle and a circular path. In order to organize the scanning's prescribed path to the electron beam gun's deflecting system, we gave the stresses that were being generated by a computer system with digital-to-analog interface realized on a digital-to-analog transformer by L-CARD and a wide-band two-channel signal amplifier. As the 
Table 3 Build-up parameters

\begin{tabular}{llll}
\hline Source power $[\mathrm{kW}]^{\mathrm{a}}$ & Displacement velocity $[\mathrm{mm} / \mathrm{sec}]$ & Wire feed rate $[\mathrm{mm} / \mathrm{sec}]$ & Wire diameter $[\mathrm{mm}]$ \\
\hline $0.9 \ldots 1.5$ & $3.5 \ldots 5.0$ & $10 \ldots 25$ & $0.8 \ldots 1.2$ \\
\hline
\end{tabular}

accelerating voltage $60 \mathrm{kV}$

experiments show, the electron beam's scanning according to these paths not only made the metal's surface clean from dirt, but also ensured a decrease in error from the wire's shake.

Figure 3 presents an external view of the finished weld beads, each of which was visually inspected for cracks and other defects. The results are given in Table 1.

\section{Numerical simulation of heat and mass transfer by the SPH method under electron-beam wire build-up} Within numerical modeling by the SPH method we accepted the following hypotheses:

1) in the process under consideration the thermal source's power, substrate feed rate $V_{s}$ and wire feed rate $V_{w}$ are constant;

2) the ambient temperature is constant;

3) the SPH method sets a thermal source in a surface layer of particles, thereby modeling an energy flow on a surface;

4) the substrate and wire materials have the same chemical composition;

5) molten metal is considered to be an incompressible Newtonian fluid whose physical parameters (density, viscosity, thermal conductivity, etc.) do not depend on temperature;

6) when describing thermal effects in the course of melting and hardening of materials, effective

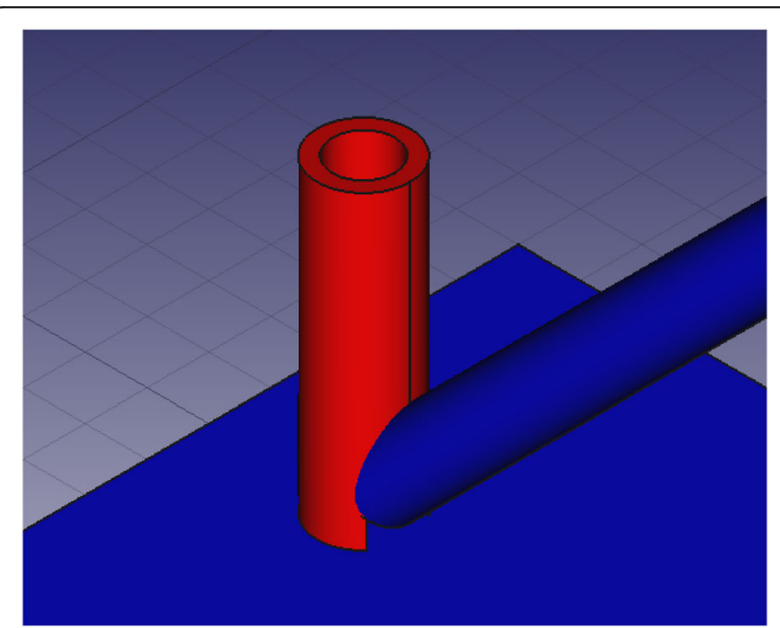

Fig. 4 The heating zone as the intersection of the cylinder (electron beam area) and the elements that identifies surface particles for setting a thermal source thermal capacity within a quasi-equilibrium model is used.

7) a two-phase zone of a melt is set by a liquidussolidus transition under melting temperature;

8) an effect of latent heat during cooling is neglected;

9) capillary forces are not taken into account;

10) an effect on the motion of a melt of electric and magnetic fields generated by an electron flow is not considered.

Table 2 shows the Grade 2 Titanium's (Tang et al. 2014; Fox and Beuth 2013) and the 308LSi Steel's (Wang et al. 2011; Tan et al. 2012; Pang et al. 2016) physical characteristics necessary for numerical modeling by the above smoothed particle method.

The wire build-up's variable parameters are presented in Table 3. In order to solve the problem, we selected various modes of the substrate's displacement and the wire's displacement with corresponding values of the thermal source's power.

The thermal source was set as follows. The LAMMPS package includes commands for geometrical primitives that allow us to single out areas of interest from the problem's geometrical model. The power source simulated the electronic beam's scanning along the circular path and was presented in the form of a hollow cylinder with external and internal diameters different from the beam's doubled diameter. The heating area was set by intersecting with the geometry elements so that the surface particles could be separated for the further setting of a thermal flow for them. Every integration step included the addition of internal energy so that a necessary heat inflow could be ensured. The particle separation by the specified method is schematically presented in Fig. 4.

\section{Results and discussion}

Preliminary numerical realizations were carried out via an multiprocessor computer (IBM 2x300 sas $15 \mathrm{k}$, 4xIntel by Xeon E7520, 64Gb) with the use of MPI multithread computations in the LAMMPS package with the following parameters:

1. wire diameter $d=0.8 \ldots 1.2 \mathrm{~mm}$;

2. substrate dimensions $10 \times 20 \times 5 \mathrm{~mm}$;

3. discretization parameter $h=0.2 \mathrm{~mm}$;

4. substrate displacement velocity $\mathrm{V}_{\mathrm{s}}=5 \mathrm{~mm} / \mathrm{sec}$;

5. wire feed rate $V_{w}=10 \ldots 15 \mathrm{~mm} / \mathrm{sec}$;

6. wire height above the substrate $H=1.5 \mathrm{~mm}$.

7. wire feed angle $\alpha=45^{\circ}$. 
Table 4 Electron-beam build-up modes

\begin{tabular}{|c|c|c|c|c|c|c|}
\hline Mode No. & Material & Power, $[\mathrm{W}]^{\mathrm{a}}$ & $\begin{array}{l}\text { Displacement } \\
\text { velocity } V_{s,}[\mathrm{~mm} / \mathrm{sec}]\end{array}$ & $\begin{array}{l}\text { Wire feed rate } \\
V_{w}[\mathrm{~mm} / \mathrm{sec}]\end{array}$ & Wire diameter, [mm] & Electronic beam scanning \\
\hline 1 & Grade 2 & 1000 & 5 & 10 & 1.2 & Circle $D=2[\mathrm{~mm}], 800[\mathrm{~Hz}]$ \\
\hline 2 & 308LSi & 1300 & 5 & 15 & 1.2 & Circle $D=2[\mathrm{~mm}], 800[\mathrm{~Hz}]$ \\
\hline 3 & $308 \mathrm{LSi}$ & 1300 & 5 & 15 & 1.2 & Circle $D=3[\mathrm{~mm}], 800[\mathrm{~Hz}]$ \\
\hline
\end{tabular}

${ }^{\mathrm{a}}$ Beam focusing on a surface took place at $\mathrm{I}=835[\mathrm{~mA}]$

With the parameters set, the problem consisted of 137,000 particles. Most particles were accounted for the substrate, therefore it is reasonable to decrease the substrate's height for the purpose of reducing a computation time, which also affects neither the heat distribution nor the build-up process.

In order to verify the computation results of the heat and mass transfer processes when building up wire materials by an electronic beam, we compared the computational and experimental results obtained when building up single beads with mode parameters varied. During the preliminary tests, we selected certain buildup modes to ensure stable formation of a bead being built-up with a continuous metal transfer from wire to the welding pool (Table 4).

Figure 5 shows the specific results of numerical modeling at various time points as exemplified in the computation for mode 1 (Table 4). These pictures show the
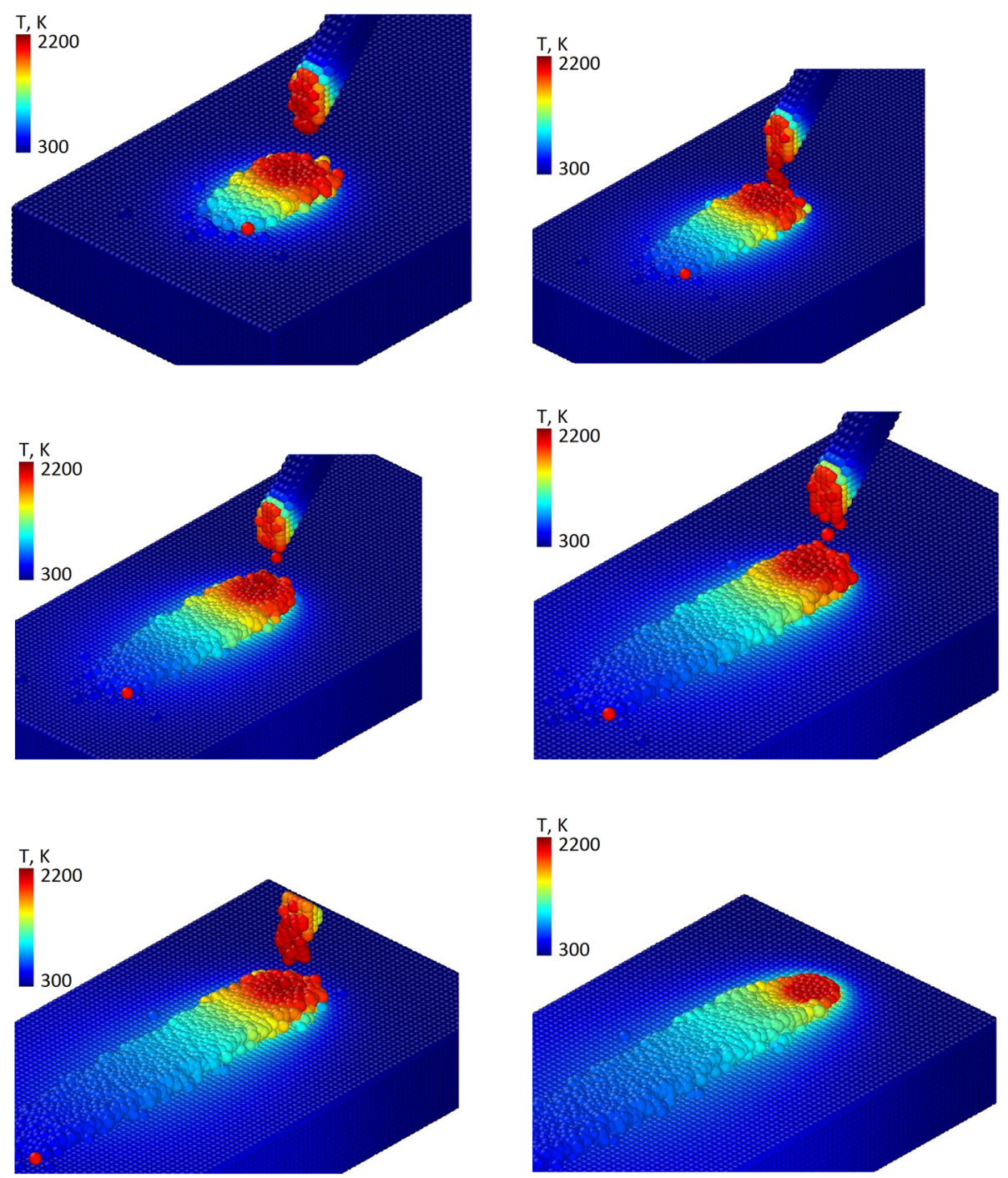

Fig. 5 Numerical modeling results at various time points at the wire rate of $10 \mathrm{~mm} / \mathrm{sec}$ and power of $1 \mathrm{KW}$ (mode 1) 


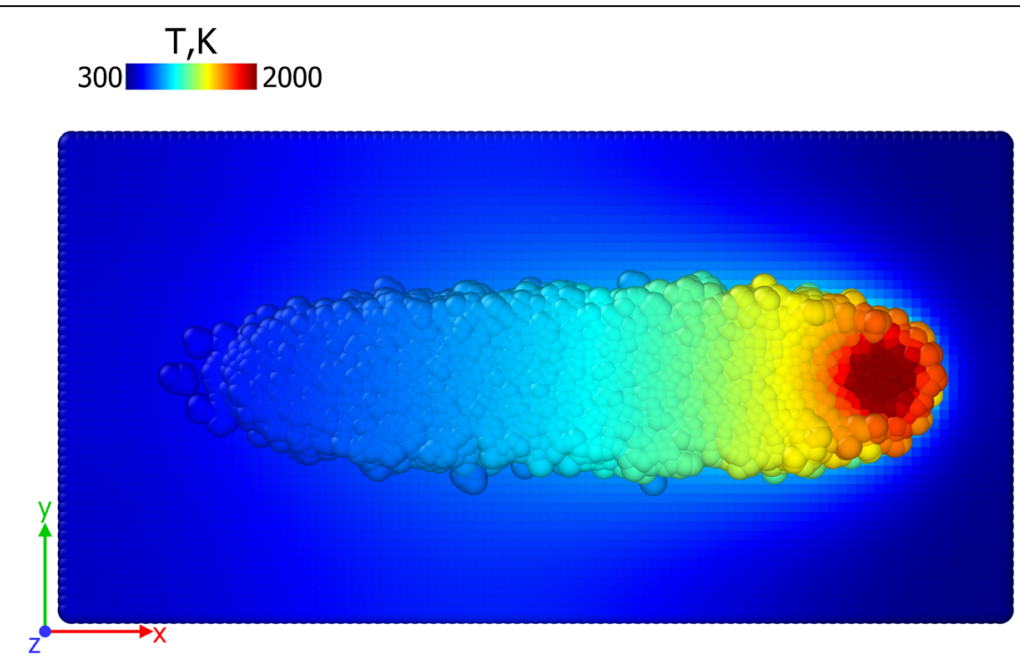

Fig. 6 The bead's structure after building up

formation of a bead from the time of thermal source's power activation and wire feed to a stable mode, then the wire feed stopped. Temperature is displayed in the color scale. As the process continues to develop, the bead's width increases and reaches an established value after $1.5 \mathrm{~s}$.

The diameter of the electronic beam's scanning affects the bead's width. The interaction of the electronic beam and the substrate's surface leads to its melting due to attacking by electrons. As a result, the best wetting takes place and the contact angle decreases.

Figure 6 presents a top view of the bead in the course of building up.

Figures 7 and 8 present the temperature distributions in the course of building up in longitudinal and cross sections.
Figure 9 shows the computational results of thermal fields and the pool's free surface when building up with steel wire (mode 2 ).

The numerical modeling results for the wire build-up under various modes were processed for the purpose of determining the bead's geometrical characteristics and the melt bath's volume. The computational values as compared to the experimental data are given in Table 1. The obtained mean-square divergence for the width $4 \%$, a maximum one is $4 \%$, for the height $-4 \%$ and $5 \%$ respectively.

\section{Conclusion}

This work presents the mathematical model that describes heat and mass transfer in the course of additive product formation by melting a wire material with a

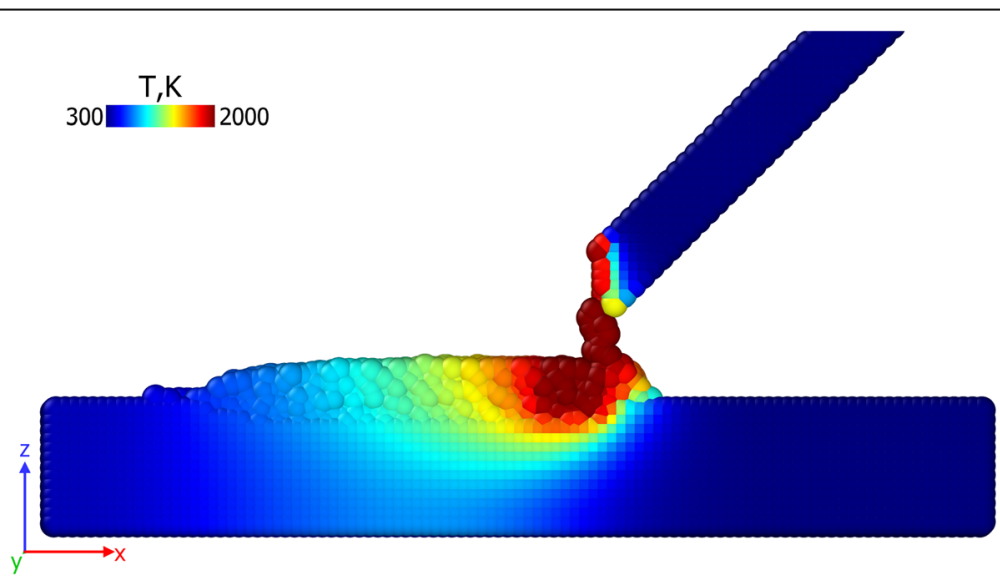

Fig. 7 The temperature distribution in the course of building up in longitudinal section 


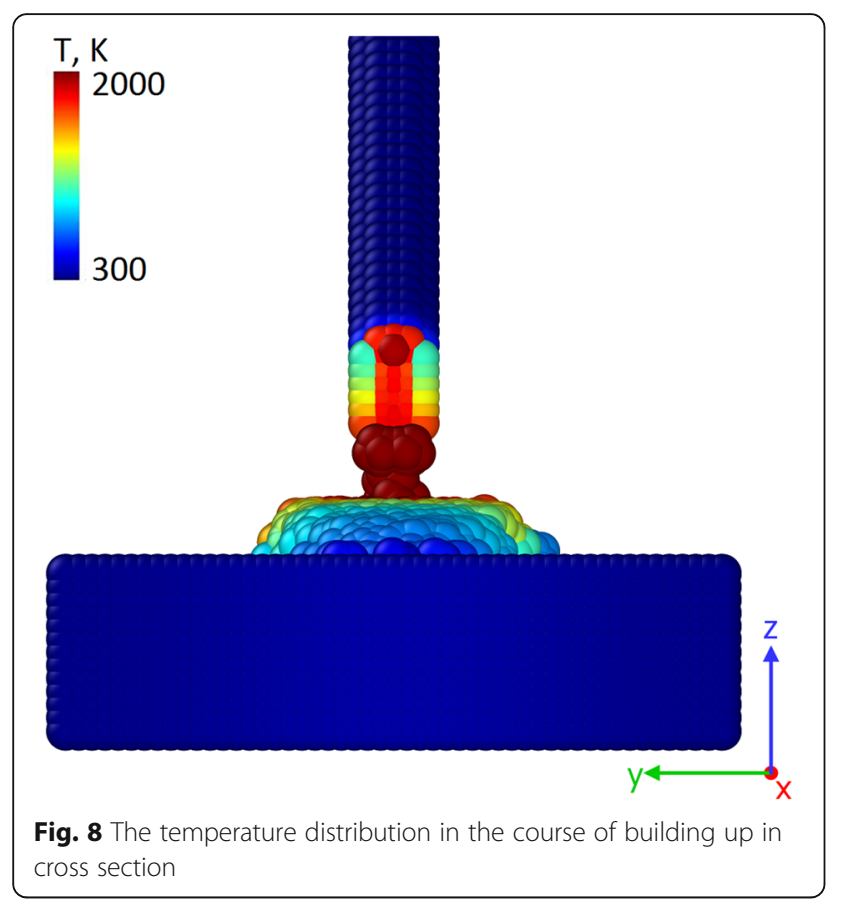

plasma (electric) arc and concentrated power sources with asymmetrical wire feed. The developed model includes:

1) non-stationary equations of heat and mass transfer;

2) considers the use of preliminary and accompanying induction heating by means of changing the initial temperature and setting the additional distributed volume thermal source;

3) thermal and hydrodynamic boundary conditions that consider the existence of a free deformable boundary of the "metal-environment" section.

The mathematical model of heat and mass transfer processes, under an additive formation process, has the following variable parameters:

1) power and type of a thermal source;

2) distribution of the density of a thermal source's energy flow;

3) initial temperature of a sample;

4) power of additional volume heating;

5) dependence of a material's thermophysical characteristics on temperature;

6) characteristics of phase transitions;

7) substrate displacement velocity;

8) wire feed rate;

9) wire height above the substrate $H=1.5 \mathrm{~mm}$;

10) wire feed angle $\alpha=45^{\circ}$.
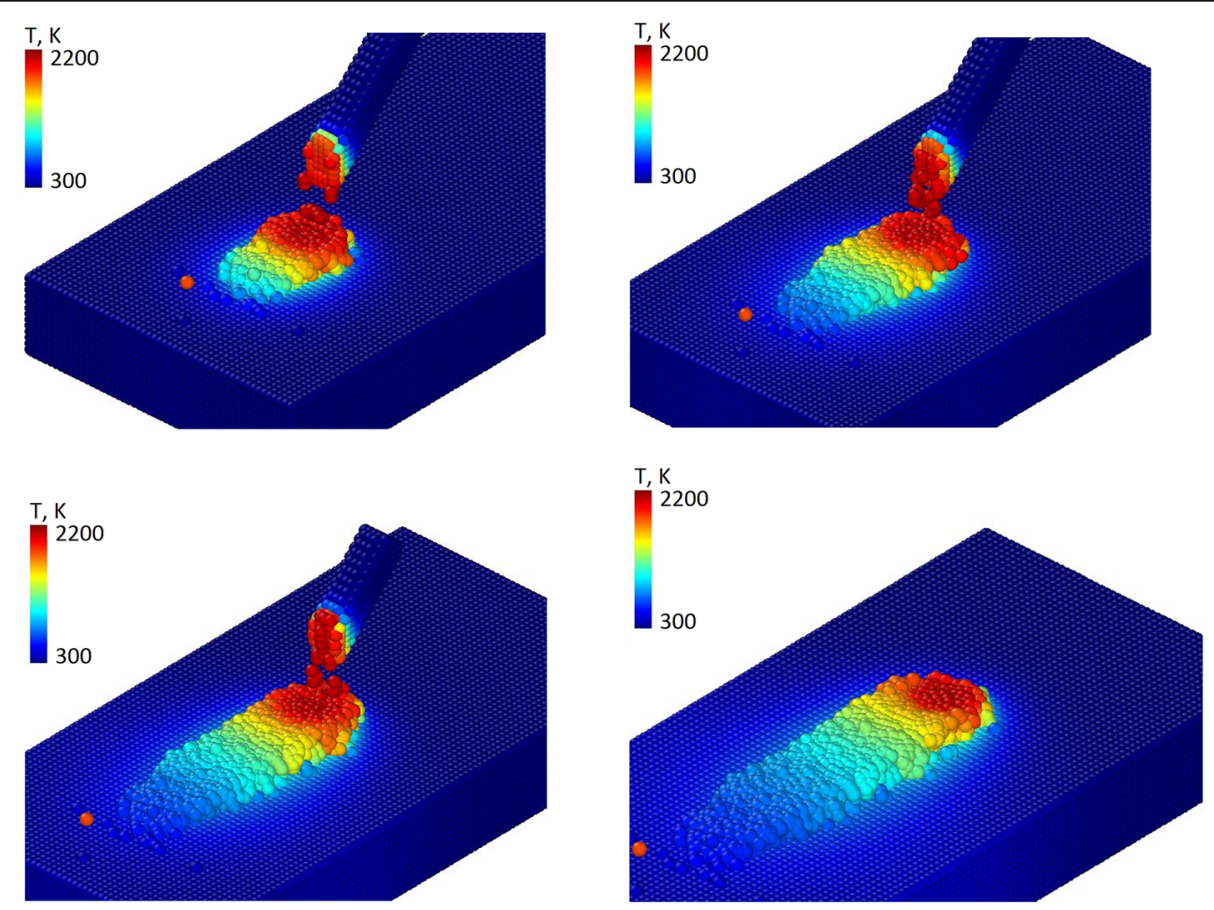

Fig. 9 The numerical modeling results at various time points at the wire rate of $15 \mathrm{~mm} / \mathrm{sec}$ and power of $1.3 \mathrm{~kW}$ (mode 2) 
In the three-dimensional formulation we implemented a mathematical model of heat and mass transfer in the process of additive formation of products by fusion of wire materials using the electron beam. The model describes non-stationary nonequilibrium conjugate heat and mass transfer processes in the molten metal with a free surface, includes differential equations of motion of a viscous medium (Navier-Stokes) and allows calculating volumetric distributions of temperatures, melt flow rates, pressures, the shape and dimensions of the molten bath, the shape of the free surface of the molten metal, the shape and dimensions of the weld bead.

The numerical implementation is carried out in a free software package for modeling LAMMPS, which also includes the implementation of the SPH method as an additional module. The proposed algorithm for solving the related heat and mass transfer problem was implemented as a script for solving the problem in the LAMMPS package. An option of setting the heat flux from the electron beam oscillating around a circle in the form of a hollow cylinder is proposed. The simulation was carried out using a high-performance computer with the MPI parallelization technology.

The multivariate modeling and verification was carried out using the example of calculating the deposition of the titanium and steel wire on the appropriate substrates for three different modes of the wire feed and heating power. The developed model allows us to predict the geometric parameters of the weld bead and make a preliminary selection of the technological parameters of the surfacing process. The error in the test examples did not exceed $10 \%$ for the height and width of the bead.

In the simulation, capillary forces were ignored due to surface tension being ignored. Despite the fact that a good agreement between the experimental and calculated data was obtained, in order to further increase the accuracy and "increase" the physicality of the model in the future works, the model will be supplemented by taking capillary forces into account.

\section{Acknowledgements}

Not applicable.

\section{Authors' contributions}

DNT - introduction, experimental setup, conclusion. EGK - experimental setup, methods, editing. RPD - algorithms, numerical simulation, methods. RMG - introduction, methods, editing. YVB - abstract, results, editing. All authors read and approved the final manuscript.

\section{Funding}

The reported study was partially supported by grants from the Russian Foundation for Basic Research RFBR №18-08-01016 A (formulation of basic tasks for the development of the process of direct metal deposition) as well as by Ministry of Education and Science of the Russian Federation at the base part of the state task No 9.9697.2017 /8.9 (review of existing technologies for direct metal deposition) and by the Government of Perm Krai research project № S-26/787 from 21.12.2017 (simulation of the process of direct metal deposition).

\section{Availability of data and materials}

The supporting data used and/or analyzed during the current study are available from the corresponding author on reasonable request.

\section{Competing interests}

The authors declare that they have no competing interests.

\section{Author details}

${ }^{1}$ Perm National Research Polytechnic University, 29 Komsomolsky prospekt, Perm, Russian Federation 614990. ${ }^{2}$ Institute of electronics, Bulgarian Academy of Sciences, 72, Tzarigradsko shose, 1784 Sofia, Bulgaria. ${ }^{3}$ University of Chemical Technology and Metallurgy - Sofia, 8, Kliment Ohridski blvd, 1756 Sofia, Bulgaria. ${ }^{4}$ Technological Center on Electron Beam and Plasma Technologies and Techniques - TC EPTT Ltd, Sofia, Bulgaria. ${ }^{5}$ Institute of Continuous Media Mechanics UB RAS, 1 Ac. Korolev str, Perm, Russian Federation 614013.

Received: 19 June 2019 Accepted: 13 November 2019

Published online: 13 December 2019

\section{References}

Alexiadis A, Ghraybeh S, Qiao G (2018) Natural convection and solidification of phase-change materials in circular pipes: a SPH approach. Comput Mater Sci 150:475-483. https://doi.org/10.1016/j.commatsci.2018.04.037

Ba K, Gakwaya A (2018) Thermomechanical total Lagrangian SPH formulation for solid mechanics in large deformation problems. Comput Methods Appl Mech Eng 342:458-473. https://doi.org/10.1016/j.cma.2018.07.038

Batra RC, Zhang GM (2004) Analysis of adiabatic shear bands in elasto-thermoviscoplastic materials by modified smoothed-particle hydrodynamics (MSPH) method. J Comput Phys 201(1):172-190. https://doi.org/10.1016/j.jcp.2004.05. 007

Batra RC, Zhang GM (2008) Modified smoothed particle hydrodynamics (MSPH) basis functions for meshless methods, and their application to axisymmetric Taylor impact test. J Comput Phys 227(3):1962-1981. https://doi.org/10.1016/ j.jcp.2007.10.001

Benz W, Asphaug E (1995) Simulations of brittle solids using smooth particle hydrodynamics. Comput Phys Commun 87(1-2):253-265. https://doi.org/10. 1016/0010-4655(94)00176-3

Bonet J, Kulasegaram S (2000) Correction and stabilization of smooth particle hydrodynamics methods with applications in metal forming simulations. Int J Numer Methods Eng 47(6):1189-1214. https://doi.org/10.1002/(SICI)10970207(20000228)47:6<1189::AID-NME830>3.0.CO;2-I

Cleary P, Ha J, Alguine V, Nguyen T (2002) Flow modelling in casting processes. Appl Math Model 26(2):171-190. https://doi.org/10.1016/S0307904X(01)00054-3

Cleary PW (2010) Extension of SPH to predict feeding, freezing and defect creation in low pressure die casting. Appl Math Model 34(11):3189-3201. https://doi.org/10.1016/j.apm.2010.02.012

Cleary PW, Das R (2008) The potential for SPH modelling of solid deformation and fracture. In: IUTAM symposium on theoretical, computational and modelling aspects of inelastic media. Springer, Dordrecht, pp 287-296. https://doi.org/10.1007/978-1-4020-9090-5_26

Demiral M (2014) SPH modeling of vibro-assisted turning of Ti alloy: influence of vibration parameters. J Vibroengineering 16(6):2685-2694 WOS: 000342905300007

Ding D, Pan Z, Cuiuri D, Li H (2015) Wire-feed additive manufacturing of metal components: technologies, developments and future interests. Int J Adv Manuf Technol 81(1-4):465-481. https://doi.org/10.1007/s00170-015-7077-3

Dong X, Li Z, Mao Z, Lin T (2018) A development of a SPH model for simulating surface Erosion by impact (s) of irregularly shaped particles. Int J Comput Methods 15(08):1850074. https://doi.org/10.1142/S0219876218500743

Dong X, Liu GR, Li Z, Zeng W (2017) Smoothed particle hydrodynamics (SPH) modeling of shot peening process. J Comput Methods Sci Eng 17(4):799825. https://doi.org/10.3233/JCM-170764

Faizal F, Septiawan RR (2014) Computational study on melting process using smoothed particle hydrodynamics. J Mod Phys 5(3):112-116. https://doi.org/ 10.4236/jmp.2014.53019

Farrokhpanah A, Bussmann M, Mostaghimi J (2017) New smoothed particle hydrodynamics (SPH) formulation for modeling heat conduction with solidification and melting. Numerical Heat Transf B Fundamentals 71(4):299312. https://doi.org/10.1080/10407790.2017.1293972 
Fathi A, Toyserkani E, Khajepour A, Durali M (2006) Prediction of melt pool depth and dilution in laser powder deposition. J Phys D Appl Phys 39(12):2613. https://doi.org/10.1088/0022-3727/39/12/022

Fortuna SV, Filippov AV, Kolubaev EA, Fortuna AS, \& Gurianov DA (2018). Wire feed electron beam additive manufacturing of metallic components. In AIP Conference Proceedings 2051(1):020092. AIP Publishing, Melville, NY. https:// doi.org/10.1063/1.5083335

Fox J, Beuth J (2013) Process mapping of transient melt pool response in wire feed e-beam additive manufacturing of Ti-6Al-4V. In: Solid freeform fabrication symposium, Austin, University of Texas, pp. 675-683

Fuchs J, Schneider C, Enzinger N (2018) Wire-based additive manufacturing using an electron beam as heat source. Welding World 62(2):267-275. https://doi. org/10.1007/s40194-017-0537-7

Ganzenmuller, G.C., Steinhauser, M.O., Van Liedekerke, P., (2011). The implementation of smooth particle hydrodynamics in LAMMPS. A guide to the SPH-USER package. https://lammps.sandia.gov/doc/PDF/SPH_LAMMPS_ userguide.pdf. Accessed 27 May 2019

Gerasimov AV, Cherepanov RO, Krektuleva RA, Barashkov VN (2017) Simulation of viscous melt drop crystallization by the SPH method. In: MATEC web of conferences 92:01071. EDP Sciences, London, UK . https://doi.org/10.1051/ matecconf/20179201071

Gingold RA, Monaghan JJ (1977) Smoothed particle hydrodynamics: theory and application to non-spherical stars. Mon Not R Astron Soc 181(3):375-389. https://doi.org/10.1093/mnras/181.3.375

Gong X, Zhang S, Yang J (2015) Elastic-plastic effect study in hypervelocity impact by SPH method. In: EPJ web of conferences 94:04059. EDP Sciences, London, UK. https://doi.org/10.1051/epjconf/20159404059

Hayhurst CJ, Clegg RA (1997) Cylindrically symmetric SPH simulations of hypervelocity impacts on thin plates. Int J Impact Eng 20(1-5):337-348. https://doi.org/10.1016/50734-743X(97)87505-7

He X, Mazumder J (2007) Transport phenomena during direct metal deposition. J Appl Phys 101(5):053113. https://doi.org/10.1063/1.2710780

Hu H, Eberhard P (2017) Thermomechanically coupled conduction mode laser welding simulations using smoothed particle hydrodynamics. Comput Part Mech 4(4):473-486. https://doi.org/10.1007/s40571-016-0140-5

Hu R, Luo M, Liu T, Liang L, Huang A, Trushnikov D et al (2019) Thermal fluid dynamics of liquid bridge transfer in laser wire deposition 3D printing. Sci Technol Weld Join:1-11. https://doi.org/10.1080/13621718. 2019.1591039

Huang Y, Khamesee MB, Toyserkani E (2016) A comprehensive analytical model for laser powder-fed additive manufacturing. Addit Manuf 12:90-99. https:// doi.org/10.1016/j.addma.2016.07.001

Jamshidinia M, Kong F, Kovacevic R (2013) Numerical modeling of heat distribution in the electron beam melting ${ }^{\circledR}$ of Ti-6Al-4V. J Manuf Sci Eng 135(6):061010. https://doi.org/10.1115/1.4025746

Jhavar S, Jain NK, Paul CP (2014) Development of micro-plasma transferred arc ( $\mu$-PTA) wire deposition process for additive layer manufacturing applications. J Mater Process Technol 214(5):1102-1110. https://doi.org/10.1016/j. jmatprotec.2013.12.016

Johnson GR, Cook WH (1985) Fracture characteristics of three metals subjected to various strains, strain rates, temperatures and pressures. Eng Fract Mech 21(1):31-48. https://doi.org/10.1016/0013-7944(85)90052-9

Johnson GR, Stryk RA, Beissel SR (1996) SPH for high velocity impact computations. Comput Methods Appl Mech Eng 139(1-4):347-373. https:// doi.org/10.1016/S0045-7825(96)01089-4

Kapil S, Legesse F, Kulkarni P, Joshi P, Desai A, Karunakaran KP (2016) Hybridlayered manufacturing using tungsten inert gas cladding. Prog Addit Manuf 1(1-2):79-91. https://doi.org/10.1007/s40964-016-0005-8

Kulasegaram S, Bonet J, Lewis RW, Profit M (2003) High pressure die casting simulation using a Lagrangian particle method. Commun Numer Methods Eng 19(9):679-687. https://doi.org/10.1002/cnm.633

Lee Y, Nordin M, Babu SS, Farson DF (2014) Effect of fluid convection on dendrite arm spacing in laser deposition. Metall Mater Trans B Process Metall Mater Process Sci 45(4):1520-1529. https://doi.org/10.1007/s11663-014-0054-7

Lee YS, Zhang W (2015) Mesoscopic simulation of heat transfer and fluid flow in laser powder bed additive manufacturing. In: International solid free form fabrication symposium, Austin, University of Texas, pp 1154-1165

Libersky LD, Petschek AG (1991) Smooth particle hydrodynamics with strength of materials. In: Advances in the free-Lagrange method including contributions on adaptive gridding and the smooth particle hydrodynamics method. Springer, Berlin, Heidelberg, pp 248-257. https://doi.org/10.1007/3-540-54960-9_58
Libersky LD, Petschek AG, Carney TC, Hipp JR, Allahdadi FA (1993) High strain Lagrangian hydrodynamics: a three-dimensional SPH code for dynamic material response. J Comput Phys 109(1):67-75. https://doi.org/10.1006/jcph.1993.1199

Liu GR (2009) Meshfree methods: moving beyond the finite element method. CRC press Boca Raton, FL 792 p. https://doi.org/10.1201/9781420082104

Liu MB, Zhang ZL, Feng DL (2017) A density-adaptive SPH method with kernel gradient correction for modeling explosive welding. Comput Mech 60(3): 513-529. https://doi.org/10.1007/s00466-017-1420-5

Lorenz KA, Jones JB, Wimpenny DI, Jackson MR (2015) A review of hybrid manufacturing. In: Solid freeform fabrication conference proceedings, vol 53, pp 96-108

Lucy LB (1977) A numerical approach to the testing of the fission hypothesis. Astron J 82:1013-1024

Ma A, Hartmaier A (2016) A crystal plasticity smooth-particle hydrodynamics approach and its application to equal-channel angular pressing simulation. Model Simul Mater Sci Eng 24(8):085011. https://doi.org/10.1088/0965-0393/ 24/8/085011

Markl M, Körner C (2016) Multiscale modeling of powder bed-based additive manufacturing. Annu Rev Mater Res 46:93-123. https://doi.org/10.1146/ annurev-matsci-070115-032158

Mladenov GM, Koleva EG, \& Trushnikov DN (2018) Mathematical modelling for energy beam additive manufacturing. In Journal of Physics: Conference Series. 1089(1):012001. IOP Publishing, Bristol, UK https://doi.org/10.1088/ 1742-6596/1089/1/012001

Monaghan JJ (2006) Smoothed particle hydrodynamic simulations of shear flow. Mon Not R Astron Soc 365(1):199-213. https://doi.org/10.1111/j.1365-2966. 2005.09704.x

Monaghan JJ, Huppert HE, Worster MG (2005) Solidification using smoothed particle hydrodynamics. J Comput Phys 206(2):684-705. https://doi.org/10. 1016/j.jcp.2004.11.039

Morris JP, Fox PJ, Zhu Y (1997) Modeling low Reynolds number incompressible flows using SPH. J Comput Phys 136(1):214-226. https://doi.org/10.1006/jcph. 1997.5776

Morville S, Carin M, Peyre P, Gharbi M, Carron D, Le Masson P, Fabbro R (2012) $2 \mathrm{D}$ longitudinal modeling of heat transfer and fluid flow during multilayered. J Laser Appl 24(3):032008 http://hdl.handle.net/10985/6858

Pang S, Shao X, Li W, Chen X, Gong S (2016) Dynamic characteristics and mechanisms of compressible metallic vapor plume behaviors in transient keyhole during deep penetration fiber laser welding. Appl Phys A Mater Sci Process 122(7):702. https://doi.org/10.1007/s00339-016-0230-5

Qi H, Mazumder J, Ki H (2006) Numerical simulation of heat transfer and fluid flow in coaxial laser cladding process for direct metal deposition. J Appl Phys 100(2):024903. https://doi.org/10.1063/1.2209807

Rahmat A, Tofighi N, Shadloo MS, Yildiz M (2014) Numerical simulation of wall bounded and electrically excited Rayleigh-Taylor instability using incompressible smoothed particle hydrodynamics. Colloids Surf A Physicochem Eng Asp 460:60-70. https://doi.org/10.1016/j.colsurfa.2014.02.044

Russell MA, Souto-Iglesias A, Zohdi TI (2018) Numerical simulation of laser fusion additive manufacturing processes using the SPH method. Comput Methods Appl Mech Eng 341:163-187. https://doi.org/10.1016/j.cma.2018.06.033

Shadloo MS, Yildiz M (2011) Numerical modeling of kelvin-Helmholtz instability using smoothed particle hydrodynamics. Int I Numer Methods Eng 87(10): 988-1006. https://doi.org/10.1002/nme.3149

Shadloo MS, Yildiz M (2012) Simulation of Rayleigh-Taylor instability by smoothed particle hydrodynamics: advantages and limitations. In AIP Conference Proceedings, 1479 (1):90-94. AlP Publishing, Melville, NY. https://doi.org/10. $1063 / 1.4756070$

Shadloo MS, Zainali A, Yildiz M (2013) Simulation of single mode Rayleigh-Taylor instability by SPH method. Comput Mech 51(5):699-715. https://doi.org/10. 1007/s00466-012-0746-2

Soylemez E, Beuth JL, \& Taminger K (2010) Controlling melt pool dimensions over a wide range of material deposition rates in electron beam additive manufacturing. In Proceedings of 21 st solid freeform fabrication symposium, Austin, University of Texas, pp. 9-11

Spreng F, Eberhard P (2018) Modeling of orthogonal metal cutting using adaptive smoothed particle hydrodynamics. In: Thermale Effects in Complex Machining Processes. Springer, Cham, pp 133-143. https://doi.org/10.1007/ 978-3-319-57120-1_8

Stawovy MT (2018) Comparison of LCAC and PM Mo deposited using Sciaky

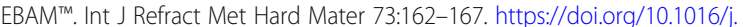
ijrmhm.2018.02.009 
Stenberg N, Delić A, Björk T (2017) Using the SPH method to easier predict wear in machining. Procedia CIRP 58:317-322. https://doi.org/10.1016/j.procir.2017.03.234

Tan W, Bailey NS, Shin YC (2012) Numerical modeling of transport phenomena and dendritic growth in laser spot conduction welding of 304 stainless steel. J Manuf Sci Eng 134(4):041010. https://doi.org/10.1115/1.4007101

Tang Q, Pang S, Chen B, Suo H, Zhou J (2014) A three dimensional transient model for heat transfer and fluid flow of weld pool during electron beam freeform fabrication of Ti-6-Al-4-V alloy. Int J Heat Mass Transf 78:203-215. https://doi.org/10.1016/j.jigheatmasstransfer.2014.06.048

Tarasov SY, Filippov AV, Savchenko NL, Fortuna SV, Rubtsov VE, Kolubaev EA, Psakhie SG (2018) Effect of heat input on phase content, crystalline lattice parameter, and residual strain in wire-feed electron beam additive manufactured 304 stainless steel. Int J Adv Manuf Technol 99(9-12):23532363. https://doi.org/10.1007/s00170-018-2643-0

Terranova B, Whittaker A, Schwer L (2018) Simulation of wind-borne missile impact using Lagrangian and smooth particle hydrodynamics formulations. Int J Impact Eng 117:1-12. https://doi.org/10.1016/j.jijmpeng.2018.02.010

Trautmann M, Hertel M, Füssel U (2018) Numerical simulation of weld pool dynamics using a SPH approach. Welding World 62(5):1013-1020. https://doi. org/10.1007/s40194-018-0615-5

Wang R, Lei Y, Shi Y (2011) Numerical simulation of transient temperature field during laser keyhole welding of 304 stainless steel sheet. Opt Laser Technol 43(4):870-873. https://doi.org/10.1016/j.optlastec.2010.10.007

Xiao Y, Han X, Hu D (2011) A coupling algorithm of finite element method and smoothed particle hydrodynamics for impact computations. Comput Mater Continua 23(1):9. https://doi.org/10.3970/cmc.2011.023.009

Xiao Y, Zhan H, Gu Y, Li Q (2017) Modeling heat transfer during friction stir welding using a meshless particle method. Int J Heat Mass Transf 104:288300. https://doi.org/10.1016/j.jheatmasstransfer.2016.08.047

Yuan P, Gu D (2015) Molten pool behaviour and its physical mechanism during selective laser melting of TiC/AISi10Mg nanocomposites: simulation and experiments. J Phys D Appl Phys 48(3):035303. https://doi.org/10.1088/00223727/48/3/035303

Zahedi SA, Demiral M, Roy A, Silberschmidt W (2013) FE/SPH modelling of orthogonal micro-machining of fcc single crystal. Comput Mater Sci 78:104109. https://doi.org/10.1016/j.commatsci.2013.05.022

Zhang Y, Bernard A, Harik R, Karunakaran KP (2017) Build orientation optimization for multi-part production in additive manufacturing. J Intell Manuf 28(6): 1393-1407. https://doi.org/10.1007/s10845-015-1057-1

Zhang ZL, Ma T, Liu MB, Feng D (2019) Numerical study on high velocity impact welding using a modified SPH method. Int J Comput Methods 16(02): 1846001. https://doi.org/10.1142/S0219876218460015

\section{Publisher's Note}

Springer Nature remains neutral with regard to jurisdictional claims in published maps and institutional affiliations.

\section{Submit your manuscript to a SpringerOpen ${ }^{\circ}$ journal and benefit from:}

- Convenient online submission

- Rigorous peer review

- Open access: articles freely available online

- High visibility within the field

- Retaining the copyright to your article

Submit your next manuscript at $\boldsymbol{\nabla}$ springeropen.com 